\title{
Momentum-space imaging of Cooper pairing in a half-Dirac-gas topological superconductor
}

\author{
Su-Yang Xu', Nasser Alidoust', llya Belopolski ${ }^{1}$, Anthony Richardella ${ }^{2}$, Chang Liu', Madhab Neupane', \\ Guang Bian', Song-Hsun Huang ${ }^{3}$, Raman Sankar ${ }^{3}$, Chen Fang ${ }^{1,4}$, Brian Dellabetta ${ }^{4}$, Wenqing Dai ${ }^{2}$, \\ Qi Li ${ }^{2}$, Matthew J. Gilbert ${ }^{4}$, Fangcheng $\mathrm{Chou}^{3}$, Nitin Samarth ${ }^{2}$ and M. Zahid Hasan ${ }^{1,5 \star}$
}

Superconductivity involving topological Dirac electrons has recently been proposed as a platform between concepts in high-energy and condensed-matter physics. It has been predicted that supersymmetry and Majorana fermions, both of which remain elusive in particle physics, may be realized through emergent particles in these particular superconducting systems. Using artificially fabricated topological-insulator-superconductor heterostructures, we present direct spectroscopic evidence for the existence of Cooper pairing in a weakly interacting half Dirac gas. Our studies reveal that two dimensional topological superconductivity in a helical Dirac gas is distinctly different from that in an ordinary two-dimensional superconductor in terms of the spin degrees of freedom of electrons. We further show that the pairing of Dirac electrons can be suppressed by timereversal symmetry-breaking impurities, thereby removing the distinction. Our demonstration and momentum-space imaging of Cooper pairing in a half-Dirac-gas two-dimensional topological superconductor serve as a critically important platform for future testing of fundamental physics predictions such as emergent supersymmetry and topological quantum criticality.

$\mathrm{R}$ ealization of novel superconductivity is one of the central themes in condensed matter physics in general ${ }^{1-24}$. Superconductivity is a collective phenomenon, where electrons moving to the opposite directions $( \pm k)$ form dynamically bound pairs, resulting in a Cooper pair gas. In an ordinary superconductor, the conduction electrons that move along a certain direction have both spin-up and spin-down electrons available for the Cooper pairing. The superconductivity observed so far, including in the conventional $s$-wave BCS superconductors as well as the cuprate or heavy fermion $d$-wave superconductors, all share this property. Recently, the discovery of 3D topological insulators (TIs) in bismuth-based semiconducting compounds has attracted much interest in condensed matter physics. In these TI materials, the bulk has a full energy gap whereas the surface exhibits an odd number of Dirac-cone electronic states, where the spin of the surface electrons is uniquely locked to their momentum ${ }^{1,2}$. Therefore, at any given surface of a TI, the surface electrons moving in one direction (for example, $+k$ ) will have only spin-up electrons available whereas those of moving to $-k$ have only spin-down electrons available. This is in contrast to the Fermi level electronic states in an ordinary superconductor. This distinction can give rise to a wide range of exotic physics. Recently, a number of theories have highlighted these possibilities from both the fundamental physics and applications point of view $^{4-10}$. For example, both supersymmetry and Majorana fermions are interesting physics phenomena predicted in high-energy theories that remain unobserved in particle physics experiments. And it has been theoretically predicted, very recently, that such new physics can be realized in a condensed matter setting ${ }^{4,6}$, if superconductivity can be induced in a spin-helical gas. Moreover, a low-energy realization of these phenomena can also be used to build the topological qubit for a topological quantum computer, which therefore is also of value in device applications. The first step towards the uambiguous realization of Majorana fermions or any of the fascinating theoretical proposals requires a clear demonstration of the helical-Cooper pairing topological superconductor phase. Helical-Cooper pairing is defined as the superconducting Bose condensation of a spin-momentum-locked Dirac electron gas, independent of the bosonic character of the pairing glue $e^{1-3}$. So far, a direct experimental demonstration of helical-Cooper pairing remains elusive.

In this paper, we use spin- and momentum-resolved photoemission spectroscopy with sufficiently high resolution and at sufficiently low temperature to allow direct evidence for the helical Cooper pairing in a spin-momentum-locked Dirac electron gas. We achieve this through the observation of the momentumresolved Bogoliubov quasi-particle spectrum of a topological insulator $\left(\mathrm{Bi}_{2} \mathrm{Se}_{3}\right)$ in proximity to a superconducting $\mathrm{NbSe}_{2}$ substrate. We further systematically investigate the dependence of the helical Cooper pairing in the Dirac electrons on varying the TI film thickness or doping magnetic impurities. Our observation of helical-Cooper pairing and superconductivity in spin-Dirac electronic gas serves as an important platform for realizing many exotic phenomena ${ }^{1-10}$. We also demonstrate a systematic methodology using the combination of spin- and momentumresolved ARPES and interface transport that can be more generally applied to discover, isolate, and systematically optimize topological superconductivity in engineered materials.

\section{Sample layout for Dirac superconductivity studies}

High-quality $\mathrm{Bi}_{2} \mathrm{Se}_{3} / 2 \mathrm{H}-\mathrm{NbSe}_{2}$ interface heterostructures (Fig. 1a,b) are prepared using molecular beam epitaxy growth

\footnotetext{
${ }^{1}$ Joseph Henry Laboratory, Department of Physics, Princeton University, Princeton, New Jersey 08544, USA, ${ }^{2}$ Department of Physics, The Pennsylvania State University, University Park, Pennsylvania 16802-6300, USA, ${ }^{3}$ Center for Condensed Matter Sciences, National Taiwan University, Taipei 10617, Taiwan, ${ }^{4}$ Department of Electrical and Computer Engineering, University of Illinois, Urbana, Illinois 61801, USA, ${ }^{5}$ Princeton Center for Complex Materials, Princeton Institute for Science and Technology of Materials, Princeton University, Princeton, New Jersey 08544, USA. *e-mail: mzhasan@princeton.edu
} 
a

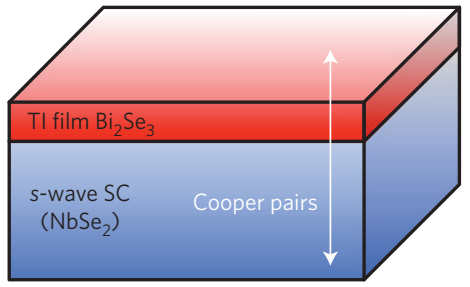

b

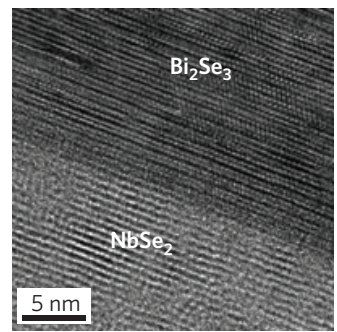

d

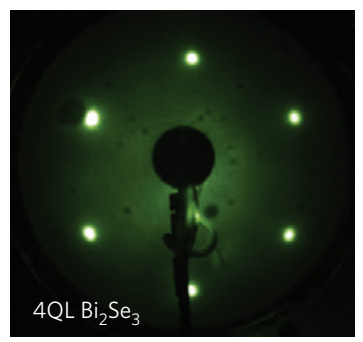

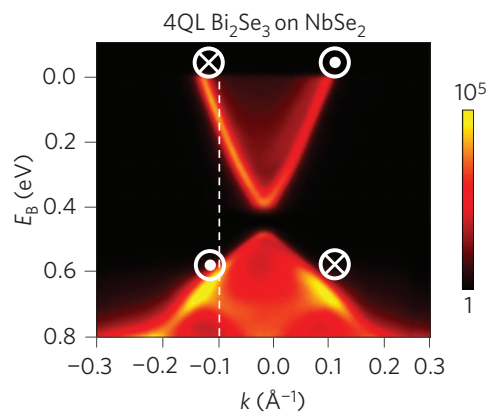

c

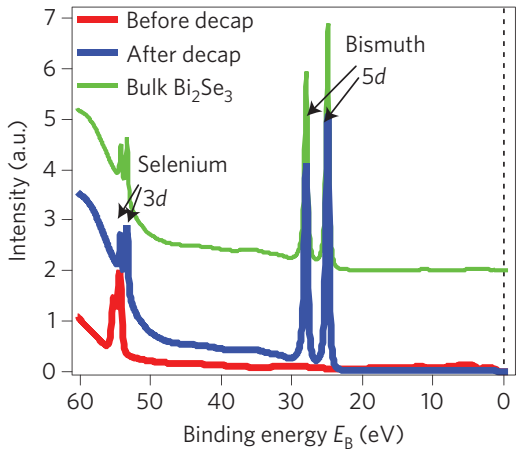

f

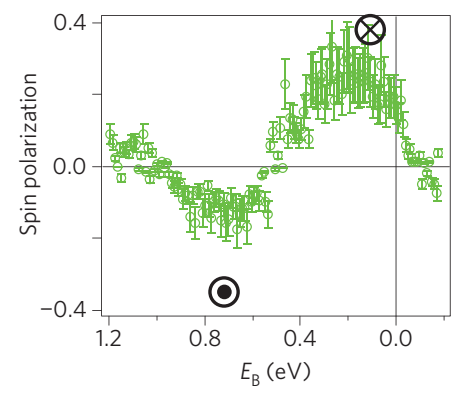

Figure 1 | Sample layout and characterization. a, A schematic layout of ultrathin $\mathrm{Bi}_{2} \mathrm{Se}_{3}$ films epitaxially grown on the (0001) surface of single-crystalline $s$-wave superconductor ( $\mathrm{SC}$ ) $2 \mathrm{H}-\mathrm{NbSe}_{2}\left(T_{\mathrm{C}}=7.2 \mathrm{~K}\right.$ ) using the MBE technique. $\mathbf{b}$, High-resolution transmission electron microscopy (TEM) measurements of the $\mathrm{Bi}_{2} \mathrm{Se}_{3} / \mathrm{NbSe}_{2}$ interface at $200 \mathrm{keV}$ electron energy. An atomically abrupt transition from the $\mathrm{NbSe}_{2}$ layered structure to the layered quintuple layer structure of $\mathrm{Bi}_{2} \mathrm{Se}_{3}$ is resolved, showing a good atomically flat interface crystal quality (see Supplementary Information $\mathrm{B}$ for details).

c, Momentum-integrated ARPES intensity curves (in arbitrary units (a.u.)) over a wide binding energy window (core-level spectra) taken on a representative $3 \mathrm{QL} \mathrm{Bi} \mathrm{Se}_{3}\left(\simeq 3 \mathrm{~nm}\right.$ ) film grown on $\mathrm{NbSe}_{2}$ before and after removing the amorphous selenium capping layer (decapping). $\mathbf{d}$, $\mathrm{A}$ low-energy electron diffraction (LEED) image on a $4 \mathrm{QL} \mathrm{Bi} 2 \mathrm{Se}_{3}$ film shows a six-fold pattern, providing evidence that the thin $\mathrm{Bi}_{2} \mathrm{Se}_{3}$ film is well ordered.

e, High-resolution ARPES dispersion map of a $4 \mathrm{QL} \mathrm{Bi}{ }_{2} \mathrm{Se}_{3}$ film on $\mathrm{NbSe}_{2}$ after decapping using an incident photon energy of 50 eV. The white circle and cross schematically show the measured direction of the spin texture on the top surface of our $4 Q \mathrm{~L} \mathrm{Bi}_{2} \mathrm{Se}_{3}$ film shown in $\mathbf{f}$. $\mathrm{A}$ colour bar is presented on the right giving the ARPES intensity on a relative scale. $\mathbf{f}$, Spin-resolved ARPES measurements on $4 \mathrm{QL} \mathrm{Bi} \mathrm{Se}_{3}$ as a function of the binding energy at a fixed momentum, which is indicated by the white dotted line in e. The error bars show the experimental uncertainty in determining the magnitude of the spin polarization.

(MBE). The growth conditions are systematically optimized to enhance the superconductivity signals in our ARPES measurements. To protect the $\mathrm{Bi}_{2} \mathrm{Se}_{3}$ surface from exposure to atmosphere, an amorphous selenium layer is deposited on top of the TI surface. This layer can be removed in situ in our angle-resolved photoemission spectroscopy (ARPES) experiments by annealing the samples, as demonstrated in Fig. 1c,d (see Supplementary Information A.1 and also ref. 25 for details). High-resolution ARPES measurements on the $\mathrm{Bi}_{2} \mathrm{Se}_{3}$ surface are then performed (Fig. 1e). A sharp spectrum for the Dirac surface states is clearly observed, indicating the good surface/interface quality of our heterostructure. Furthermore, we perform spin-resolved ARPES measurements (photon energy $50 \mathrm{eV}$ ) on the 4-quintuple-layer (QL) sample (Fig. 1f). At the Fermi level, a left-handed spin-momentum-locking profile is observed, which is one of the critical ingredients for the helical-Cooper pairing, as we will show in later sections. In Supplementary Information $\mathrm{C}$, we further present comprehensive spin-resolved measurements and demonstrate reliability.

\section{Pairing gap in helical surface states}

Figure 2a shows the ARPES dispersion maps of the $\mathrm{Bi}_{2} \mathrm{Se}_{3}$ films with different thicknesses grown on $\mathrm{NbSe}_{2}$. We start with the 4QL sample using an incident photon energy of $18 \mathrm{eV}$, as shown in Fig. $2 \mathrm{~b}$. Six representative momenta, namely $\pm \hbar k_{1}, \pm \hbar k_{2}$ and $\pm \hbar k_{3}$ are chosen for detailed studies, where $k_{1}=0.12 \AA^{-1}$ corresponds to the topological surface states (TSSs), and $k_{2}=0.08 \AA^{-1}$ and $k_{3}=0.04 \AA^{-1}$ correspond to the outer and inner parts of the bulk band states, respectively. Figure $2 \mathrm{c}$ shows the ARPES spectra at a momentum of $k_{1}$ (TSSs) at different temperatures. Clear leading-edge shifts (superconducting gap) and coherence peaks are observed at low temperatures. The observed superconducting signals as temperature increases disappear at higher temperatures such as $T=7 \mathrm{~K}$ and $12 \mathrm{~K}$. To better visualize the superconductivity gap in our data, the ARPES spectra are symmetrized with respect to the Fermi level, where the temperature evolution of the full (symmetrized) superconducting gap and the coherence peaks are clearly seen in Fig. 2d. These measurements show the existence of induced superconductivity in the helical Dirac electrons occurring in the $\mathrm{Bi}_{2} \mathrm{Se}_{3}$ TSSs, which is not possible in conventional momentum-integrated experiments that lack spin resolution ${ }^{11-20}$. We compare and contrast the proximityinduced superconductivity in the Dirac surface states to that of the bulk band states, as shown in Fig. 2e. To obtain the magnitude of the superconducting energy gap (shown in Fig. 2f), we fit the bulk state $\left( \pm \hbar k_{2}\right.$, and $\left.\pm \hbar k_{3}\right)$ data by the Dynes function ${ }^{26}$ (black curves in Fig. $2 \mathrm{~g}$ ), which is widely used in $s$-wave superconductors, whereas the surface state data $\left( \pm \hbar k_{1}\right)$ is fitted by a BCS function taking into account the spin-momentum-locking and Dirac dispersion properties of the TSSs (blue curves in Fig. $2 \mathrm{~g}$ ). To further isolate the signals of Dirac surface states from the bulk bands, we choose another incident photon energy of $50 \mathrm{eV}$ (Fig. 2h), where we use the photoemission matrix element effect to suppress the spectral weight of the bulk conduction states. We subsequently study the spectra at momentum $k_{1}$ where the ARPES signal is dominated by the contribution from the surface states. Leading-edge shifts 
a

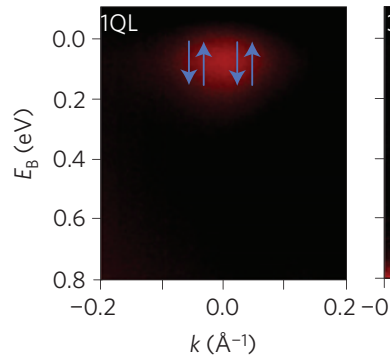

b

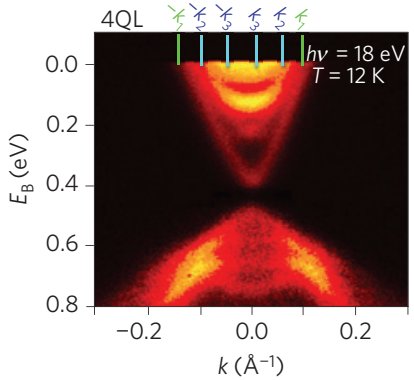

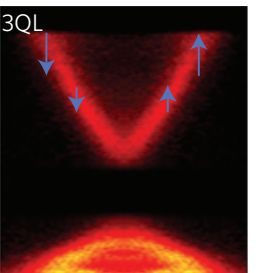

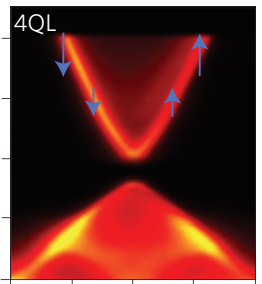

$k\left(\AA^{-1}\right)$
C

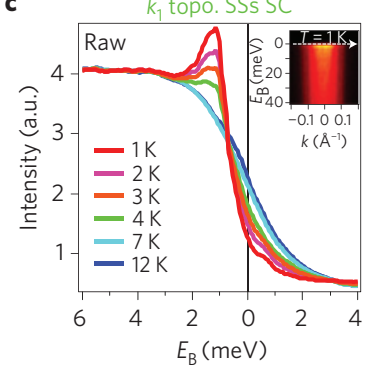

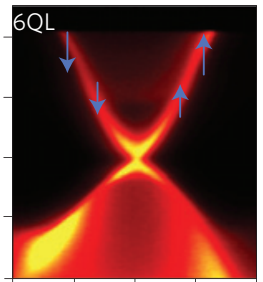

$0.2-0.2$

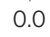

$k\left(\AA^{-1}\right)$

d

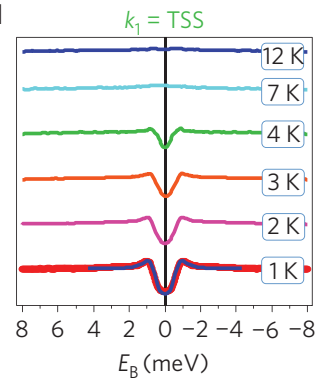

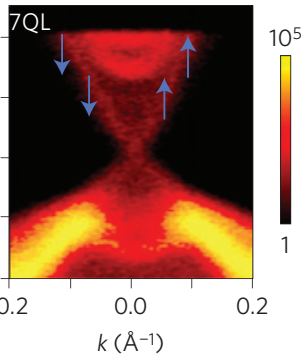

e

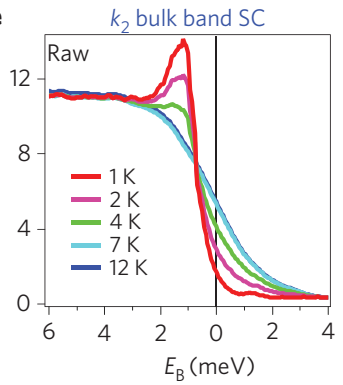

f

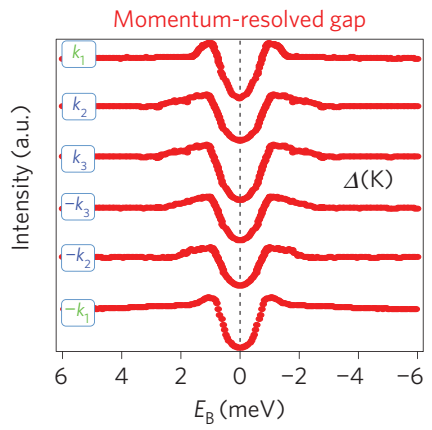

g

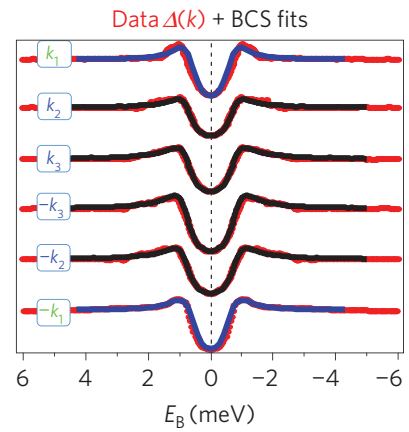

h

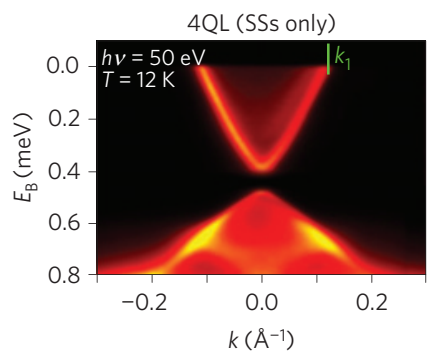

i

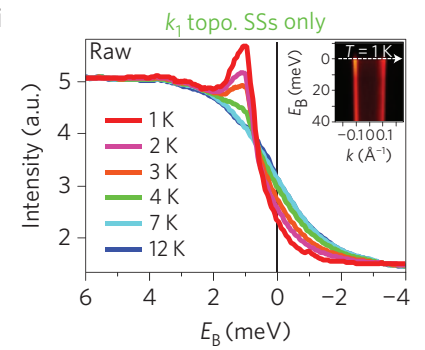

j

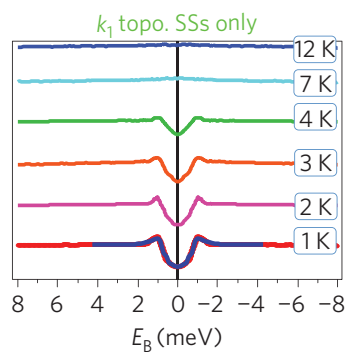

Figure 2 | Spectroscopically resolved proximity-induced topological superconductivity. a, ARPES dispersion maps of $\mathrm{Bi}_{2} \mathrm{Se}_{3} / \mathrm{NbSe}_{2}$ as a function of $\mathrm{Bi}_{2} \mathrm{Se}_{3}$ film thickness. The blue arrows quantitatively depict the spin texture configuration in the ultrathin limit. The length of the arrow is proportional to the magnitude of the spin polarization. $\mathbf{b}$, ARPES dispersion map of a $4 \mathrm{QL} \mathrm{Bi} \mathrm{Se}_{3}$ film measured at $T=12 \mathrm{~K}$ using an incident photon energy of $18 \mathrm{eV}$. c, ARPES spectra at fixed momentum $k_{1}$ (the topological surface states). d, Symmetrized ARPES spectra at $k_{1}$. e, ARPES spectra at fixed momentum $k_{2}$ (bulk band states). $\mathbf{f}, \mathbf{g}$, Symmetrized ARPES spectra at $\pm k_{1}, \pm k_{2}$ and $\pm k_{3}$ at $T \sim 1 \mathrm{~K}(\mathbf{f})$. The surface state gap ( $\pm k_{1}$ ) is further fitted (blue curves) by a BCS function taking into account its spin-momentum-locking and Dirac dispersion properties (Supplementary Information A), whereas the bulk gap is fitted (black curves) by the Dynes function ${ }^{26}$ (g). h. ARPES dispersion map of a $4 \mathrm{QL} \mathrm{Bi} 2 \mathrm{Se}_{3}$ film measured with an incident photon energy of $50 \mathrm{eV}$. i,j, ARPES spectra (i) and symmetrized ARPES spectra (j) at fixed momentum $k_{1}$. The insets of $\mathbf{c}, \mathbf{i}$ show an ARPES dispersion map near the Fermi level at a low temperature of $T \sim 1 \mathrm{~K}$ at photon energies of $18 \mathrm{eV}$ and $50 \mathrm{eV}$, respectively.

and coherence peaks (Fig. 2i,j) are clearly observed from $k_{1}$, which confirms the superconductivity in the Dirac surface states using a different photon energy. In Supplementary Information F.4.1, we present additional data and analysis to further exclude extrinsic possibilities or artefacts.

We perform ARPES measurements around the surface state Fermi surface as a function of Fermi surface azimuthal angle $\theta$, to study the extent of anisotropy of the surface state superconducting gap. Five representative momentum-space cut-directions $\left(\theta_{1}-\theta_{5}\right)$ are chosen, as indicated by the dotted lines in Fig. 3a. The helicalsurface state superconducting gap observed by ARPES at different $\theta$ angles and their fits are shown in Fig. 3b. The reasonably good surface state fitting results (blue curves in Figs $2 \mathrm{~g}$ and $3 \mathrm{~b}$ ) indicate that the obtained surface state superconductivity is consistent with its spin-helical and linear dispersive properties, which supports its helical-Cooper pairing nature. The obtained magnitude of the superconducting order parameter is then plotted as a function of Fermi surface angle in Fig. 3c. The superconducting gap is found to be nearly isotropic, which is also consistent with the time-reversalinvariant helical nature of the surface state superconductivity as expected theoretically ${ }^{6,7}$. We note that although helical Cooper pairing can also occur in other $2 \mathrm{D}$ systems with spin-polarized 
a

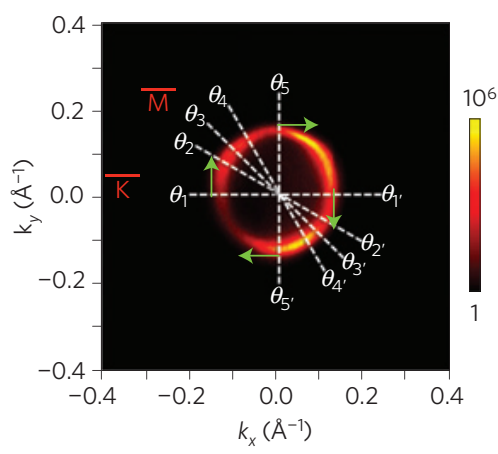

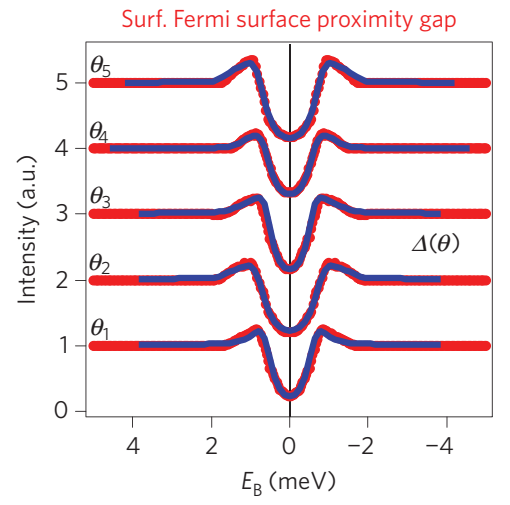

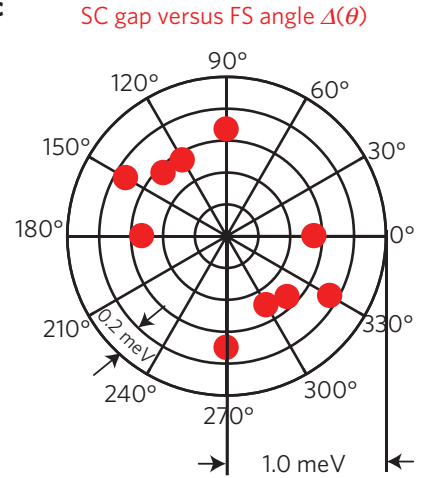

d

Topological superconducto helical Cooper pairing in TI

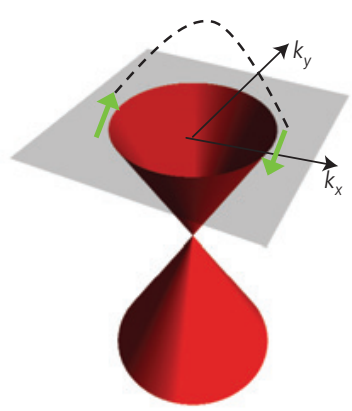

e

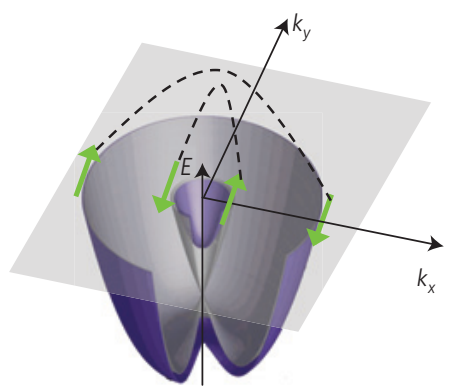

f

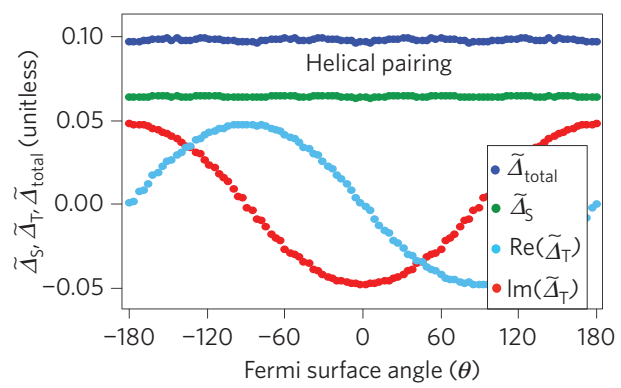

Figure 3 | Topological superconducting gap and helical Cooper pairing. a, Fermi surface map taken at an incident photon energy of $50 \mathrm{eV}$. The white dotted lines indicate the momentum-space cut-directions chosen to study the surface state superconducting gap as a function of Fermi surface angle $\theta$ around the surface state Fermi surface. b, Symmetrized and normalized ARPES spectra along $\theta_{1}$ through $\theta_{5}$ respectively (red) and their surface gap fittings. c, Fermi surface angle dependence of the estimated superconducting gap around the surface state Fermi surface. d, Illustrations depicting helical-Cooper pairing in a spin-momentum-locked helical electron gas. There is only one (an odd number of) helical Cooper pairing channel(s) for the single Dirac-cone surface states per Brillouin zone in a TI. e, Helical Cooper pairing can also occur in other 2D systems with spin-polarized bands, such as a Rashba 2DEG, but a key distinction is that in a Rashba 2DEG there are two (an even number of) channels for helical Cooper pairing per Brillouin zone (the helical Cooper pairing within the inner and the outer Fermi surface, respectively). The arrows in d,e show the direction of the spin polarization of the electrons at the Fermi level. f, Model calculation results of a topological insulator film in proximity to an s-wave superconductor (see Supplementary Information D.2 for more details) show the calculated total superconducting pairing amplitude and its decomposed singlet $\left(\widetilde{\Delta}_{\mathrm{S}}\right)$ and triplet ( $\widetilde{\Delta}_{\mathrm{T}}$ ) components on the top surface of a four-unit-cell-thick TI interfaced with an s-wave superconductor, which further confirms the helical (topologically nontrivial) nature of the induced surface state superconductivity in our $\mathrm{Bi}_{2} \mathrm{Se}_{3}$ films. Because $\widetilde{\Delta}$ is a dimensionless number (see Supplementary Information $\mathrm{D}$ for discussion), we use a tilde in $\widetilde{\Delta}$ to differentiate it from the superconducting gap $\Delta$ measured in the experiments.

bands, such as a Rashba 2DEG, a key distinction is that in a Rashba 2DEG there are two channels (an even number) for helical Cooper pairing per Brillouin zone (the helical Cooper pairing within the inner and the outer Fermi surfaces, respectively, see Fig. 3e). In contrast, in the system considered in this work, there is only one channel (an odd number) for helical Cooper pairing (the single channel provided by the Dirac surface states, Fig. 3d). The odd number of helical Cooper pairing channels, as demonstrated here, is indispensable for many fascinating phenomena predicted in theor $y^{4-10}$. In Fig. 3f and Supplementary Information D, we present model calculation analysis, which shows a $p_{x} \pm i p_{y}$ superconducting order parameter in the Dirac surface states, supporting the helical Cooper pairing nature. Therefore, through our systematic ARPES measurements with simultaneous energy, momentum and spin resolution, we observe superconductivity in an odd number of spin-momentum-locked Dirac surface states, which serves as direct experimental evidence for helical-Cooper pairing.

\section{Surface-surface hybridization and magnetic doping}

We study the observed superconductivity in the surface states as a function of surface-to-surface hybridization strength (effectively as a function of the TI film thickness), to experimentally prove its proximity-induced nature. As shown in Fig. 4a, a sample with a Dirac point hybridization gap as large as $\sim 200 \mathrm{meV}$ is realized in a $3 \mathrm{QL}$ film sample. Clear leading-edge shifts, coherence peaks and their temperature evolution are observed in the TSSs near the Fermi energy (Fig. 4b,c), evidence for helical-Cooper pairing and helical superconductivity in the $3 \mathrm{QL}$ film samples. The observed surface state superconducting gap value is about $0.7-0.8 \mathrm{meV}$. We now turn to a gapless (7QL) sample, as shown in Fig. $4 \mathrm{~d}$. The absence of hybridization gap at the Dirac point reveals that surface state wavefunctions from the top and the interface surfaces are completely separated in real space $e^{25,27,28}$. Finite superconductivity signals are observed in the ARPES spectra both at momenta of $k_{1}$ (Dirac surface states) and $k_{2}$ (bulk conduction states), which are found to be weaker than the gapped samples (Fig. 4e,f). The $\mathrm{Bi}_{2} \mathrm{Se}_{3}$ top surface's superconducting gap as a function of Dirac point gap value (surface-to-surface hybridization strength) is shown in Fig. 4g,h in a (surface and bulk) band-resolved fashion. It can be seen that the induced superconducting gap near the top surface increases with a larger hybridization gap, which is realized in thinner TI films. This observation is qualitatively consistent with the theoretical description of the superconducting proximity effect, where the Cooper pair potential on the top surface is 


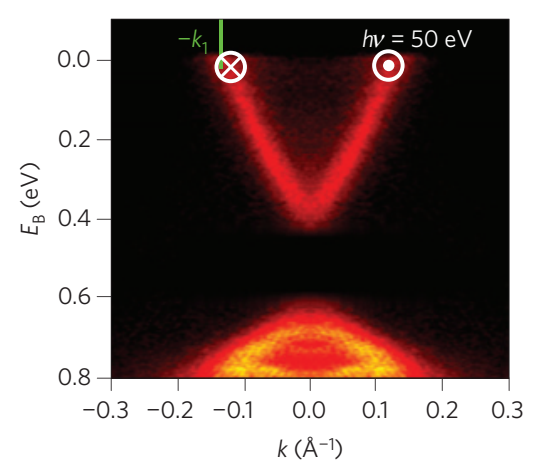

d

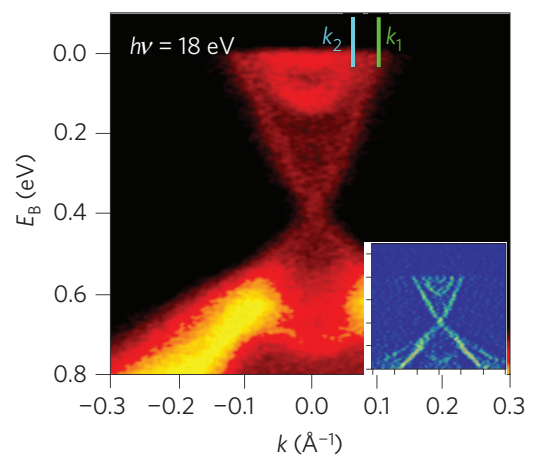

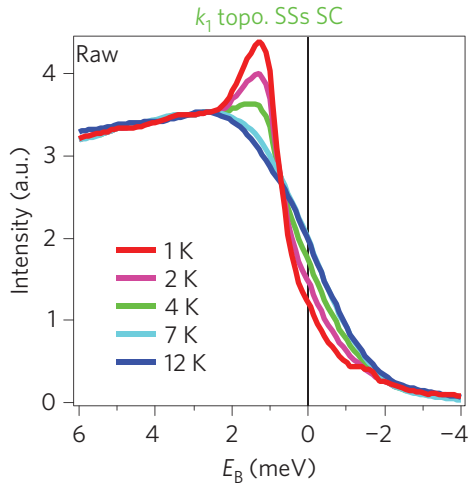

e

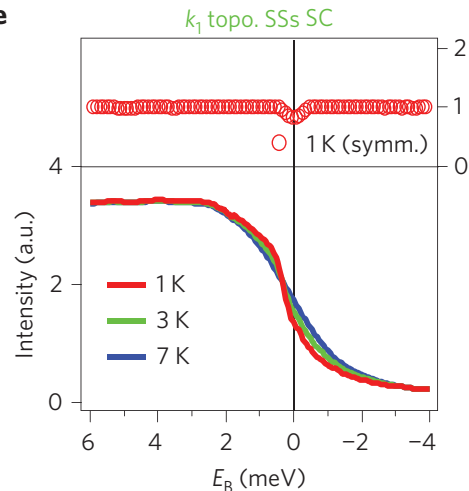

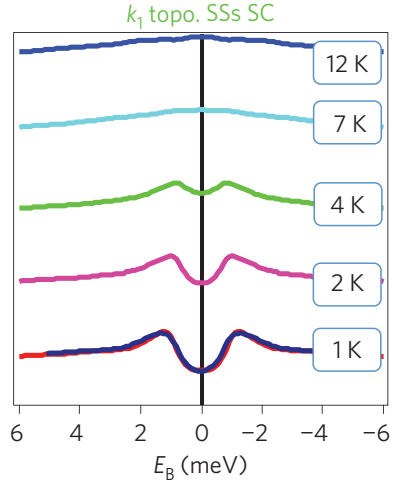

f

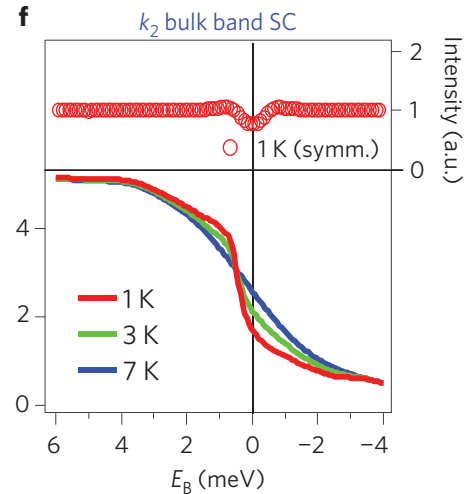

g

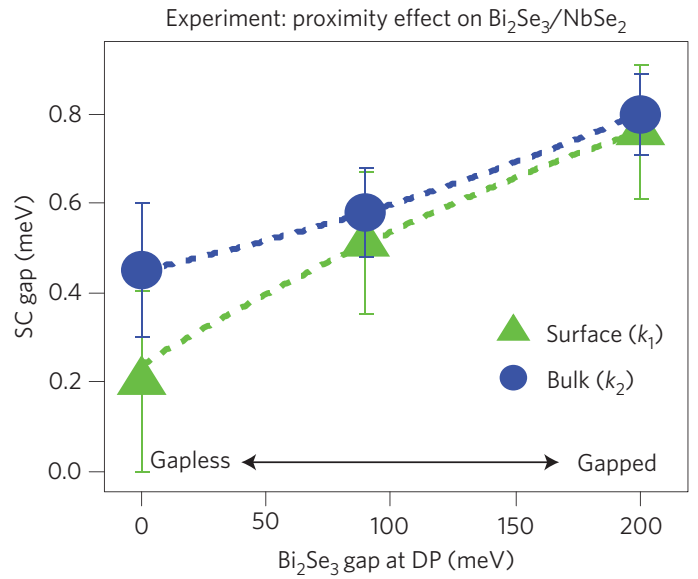

h

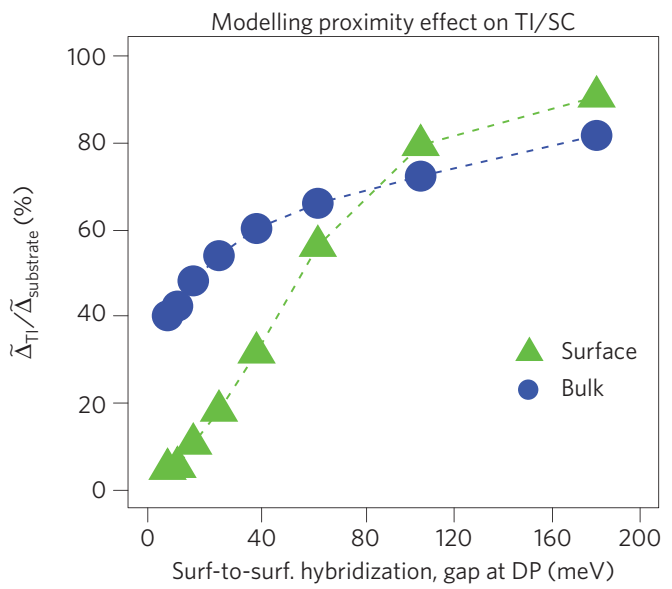

Figure 4 | Hybridization dependence of the superconducting gap. a, ARPES dispersion map of a $3 \mathrm{QL} \mathrm{Bi}_{2} \mathrm{Se}_{3}$ film measured at $T=12 \mathrm{~K}$ using an incident photon energy of $50 \mathrm{eV}$. b, ARPES spectra at fixed momentum $k_{1}$ at different temperatures. $\mathbf{c}$, Symmetrized ARPES spectra at different temperatures. d, ARPES dispersion map of a $7 \mathrm{QL} \mathrm{Bi}{ }_{2} \mathrm{Se}_{3}$ film measured at $T=12 \mathrm{~K}$ using an incident photon energy of $18 \mathrm{eV}$. The inset shows a second derivative image of the ARPES dispersion map. e,f, ARPES spectra at fixed momenta $k_{1}$ (surface states (e)) and $k_{2}$ (bulk states (f)). g, ARPES-measured superconducting gap for topological surface states $\left(k_{1}\right)$ and for the bulk conduction states $\left(k_{2}\right)$ as a function of the Dirac point gap value (surface-to-surface hybridization strength). The dotted lines are guides to the eye. The error bars here show the uncertainty in determining the superconducting gap via fitting the ARPES spectra. $\mathbf{h}$, Calculated dependence on surface-to-surface hybridization. Because the pairing amplitude $\widetilde{\Delta}_{T /}$ in the calculation is a dimensionless number, we normalize it by the pairing amplitude of the substrate superconductor, $\widetilde{\Delta}_{\text {substrate }}$ (a constant).

enhanced with the decreasing thickness of the normal metal. More interestingly, it can be seen that the surface state superconducting gap increases at a faster rate than that of the proximity gap on the bulk band. Such a contrast reveals that stronger surface-tosurface hybridization significantly enhances the helical pairing in the surface states on the top surface. These fine details of the superconducting proximity effect observed in our data will be a valuable guide in properly interpreting the vast complexity of the transport data addressing the proximity effects in TI films and heterostructures. In Supplementary Information A.5, we further present our ARPES data on magnetically $(\mathrm{Mn})$ doped $\mathrm{Bi}_{2} \mathrm{Se}_{3}$ grown on top of $\mathrm{NbSe}_{2}$, where we show that magnetic impurities lead to strong pair-breaking in both conventional (bulk) and helical (surface) pairing channels. By showing that the superconducting gap in the surface states decreases in the presence of magnetic dopants (Supplementary Information A.5), we have provided an important check for our main result that we have observed Cooper pairing in the surface states. Therefore, our measurements have 
a

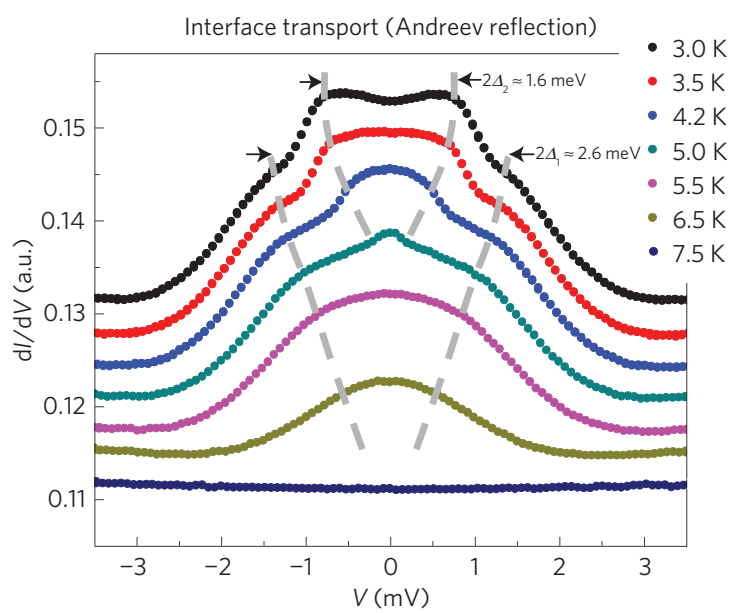

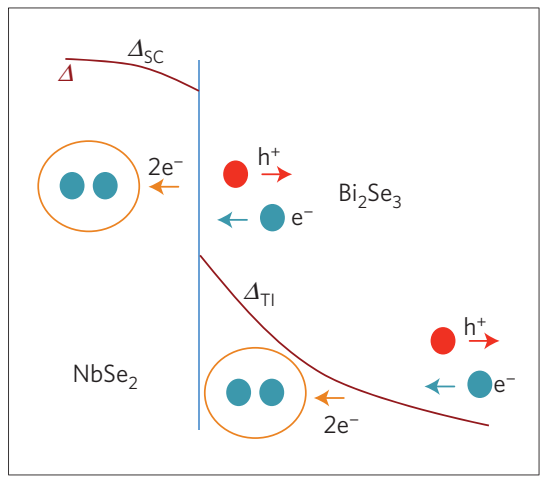

c

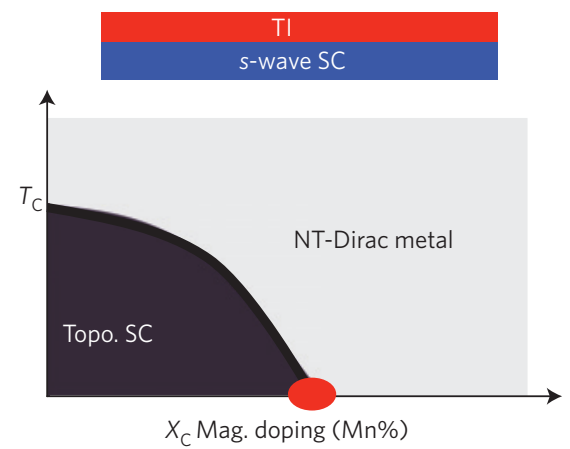

d

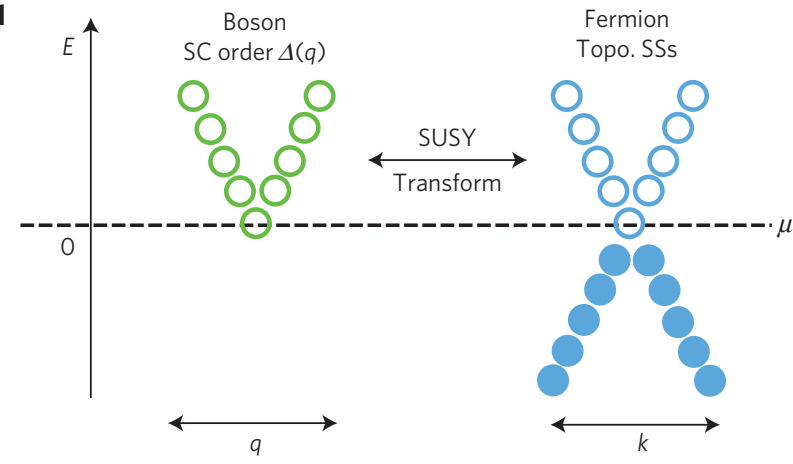

Figure 5 | Point-contact interface transport and conditions for predicted emergent supersymmetry. a, Point-contact transport ( $d / / d V$ versus bias voltage) as a function of temperature. For $T=3.5 \mathrm{~K}$, below the $T_{c}$ of $\mathrm{NbSe}_{2}$, as the bias voltage is swept from $\pm 3 \mathrm{mV}$ to $0 \mathrm{mV}$, dl/dV first increases, then levels off at $|V| \simeq 1.3 \mathrm{mV}$, and then increases again, reaching a maximum at $|\mathrm{V}| \simeq 0.8 \mathrm{mV}$. $\mathrm{dl} / \mathrm{d} V$ for other $T<T_{\mathrm{c}}$ exhibits a similar behaviour. This indicates two Andreev-reflection channels with different sizes of superconducting gap. $\mathbf{b}$, The two Andreev-reflection processes via the induced superconducting gap in $\mathrm{Bi}_{2} \mathrm{Se}_{3}$ and intrinsic superconducting gap in $\mathrm{NbSe}_{2}$, respectively. $\mathrm{e}^{-}$and $\mathrm{h}^{+}$represent electrons and holes that are involved in the Andreev-reflection process. c,d, Theoretically proposed ${ }^{4}$ unusual criticality related to supersymmetry phenomena can be realized in the topological insulator/s-wave superconductor interface as the chemical potential is tuned to the surface state Dirac point and an in-plane magnetization drives the system to the critical point between superconducting and normal non-topological (NT) Dirac metal phases (schematically shown in c). Fermions and bosons are expected to feature the same band velocity and quasi-particle lifetime $\mathrm{e}^{4}$ (schematically shown in $\mathbf{d}$ ), where the fermion velocity is estimated to be around $5 \times 10^{5} \mathrm{~m} \mathrm{~s}{ }^{-1}$ from our ARPES result. This cartoon refers to a future possibility.

identified two ways to suppress the helical Cooper pairing near the top surface of the $\mathrm{Bi}_{2} \mathrm{Se}_{3}$ film away from the TI/SC interface: increasing film thickness or doping with time-reversal-breaking magnetic elements.

\section{Point-contact Andreev-reflection transport}

To check that the top surface superconductivity is indeed a proximity effect, we use the well-established methodology of point-contact Andreev-reflection measurements to compare the induced gap values at the $\mathrm{NbSe}_{2} / \mathrm{Bi}_{2} \mathrm{Se}_{3}$ heterointerface. The point contact probe is characterized by measurements on pure $\mathrm{NbSe}_{2}$ (ref. 29) (shown in Supplementary Information E.1). The pointcontact spectra on a $\mathrm{NbSe}_{2} / \mathrm{Bi}_{2} \mathrm{Se}_{3}(16 \mathrm{QL})$ heterostructure sample are shown in Fig. 5a,b. At temperatures below the $T_{\mathrm{c}}(\sim 7.2 \mathrm{~K})$ of $\mathrm{NbSe}_{2}$, the differential conductance $(\mathrm{d} I / \mathrm{d} V)$ around zero bias increases as a result of the Andreev-reflection process, similar to the bare $\mathrm{NbSe}_{2}$ spectra. Interestingly, a second differential conductance increase appears below $\sim 5 \mathrm{~K}$. The sharp rise of differential conductance corresponds to the energy gap of the superconducting layer. From the data, we obtain the larger gap $\left(\Delta_{1}\right)$ changing from 0 to $1.3 \pm 0.2 \mathrm{mV}$ from $7.5 \mathrm{~K}$ to $3.0 \mathrm{~K}$. The second gap $\left(\Delta_{2}\right)$ feature changes from 0 to $0.8 \pm 0.2 \mathrm{mV}$ from $5.0 \mathrm{~K}$ to $3.0 \mathrm{~K}$ (See Supplementary Information E.2 for more details). Injected electrons from the point-contact are Andreev-reflected inside the superconducting $\mathrm{Bi}_{2} \mathrm{Se}_{3}$ proximity layer if their energies are lower than the induced superconducting gap in $\mathrm{Bi}_{2} \mathrm{Se}_{3}$. When their energies are above the induced gap $\Delta_{\mathrm{TI}}$ but below the $\mathrm{NbSe}_{2}$ superconducting gap $\Delta_{\mathrm{SC}}$, injected electrons are not affected by the order parameter in the $\mathrm{Bi}_{2} \mathrm{Se}_{3}$ layer but Andreev-reflected in the $\mathrm{NbSe}_{2}$ region. Therefore, the edge around $\Delta_{1}$ is probably due to the $\mathrm{NbSe}_{2}$ gap, whereas the sharp edge around $\Delta_{2}$ in the conductance spectrum probably reflects the induced gap in $\mathrm{Bi}_{2} \mathrm{Se}_{3}$ near the interface. It is worth noting that, unlike ARPES, which is mostly sensitive to the top surface, the point-contact transport probes deeper into the superconductor, similar to the electron mean free path, which is estimated to be $\sim 16 \mathrm{~nm}$ in our films (see Supplementary Information E.2 for more details). Our results thus suggest that the combination of ARPES and point-contact transport together provides a powerful method for probing superconducting proximity effects which can be used to correlate the proximity gap on the top surface and the buried interface if film thickness is not too large (not larger than the superconducting coherence length). 


\section{Discussions}

In contrast to idealized theoretical models $s^{6,7}$ of topological superconductivity where only Dirac surface states cross the Fermi level, real samples exhibit a complex phenomenology due to the coexistence of multiple bands at the chemical potential, as demonstrated in our data above. Thus, the interpretation of experimental studies must take into account both the desirable Cooper pairing from the Dirac surface states and conventional superconductivity from the bulk, trivial surface states and impurity surface states. The coexistence of multiple bands at the Fermi level means that any superconductivity realized in actual TI materials consists of not only the desirable helical Cooper pairing from the Dirac surface states, but also conventional superconductivity from the bulk states, as shown above in our data. We note that, although progress has been reported by using conventional transport and STM experiments ${ }^{11-20}$, those studies do not have the spin and momentum resolution necessary to distinguish helical Cooper pairing from that of the conventional superconductivity from other bands that intermix at the interface, making the interpretation of Majorana fermions unreliable or complex. Hence a direct experimental demonstration of the existence of superconductivity in the helical Cooper pairing channel remained elusive before our momentum and spin space observations reported here. In fact, it has been recently shown, both theoretically and experimentally ${ }^{21-24}$, that the conventional superconductivity in the bulk and impurity bands at the interface or surface lead to ambiguous interpretations of the transport and STM data. To achieve a clear case for a Majorana zero mode, the helical component of the Cooper pairing must be isolated, as demonstrated here. Therefore, it is in this context that our observation of helical Cooper pairing and superconductivity in a half Dirac gas is of critical importance. Furthermore, the overall methodology employed here can be applied to isolate helical Cooper pairing in other systems and, in connection to a feedback loop, for material growth for the optimization of the helical channel. We also note that our systematic studies (by observing the superconducting gap (leading-edge shift), the clear coherence peak, as well as their systematic dependence on varying temperature, TI film thickness and doping magnetic impurities) identify the helical Cooper pairing and topological superconductivity in the TI Dirac surface states. This is in contrast to the ARPES results on $\mathrm{Bi}_{2} \mathrm{Se}_{3} / \mathrm{BSCCO}$ samples ${ }^{30-32}$. Thus, our data strongly supports the view that $\mathrm{TI} / \mathrm{NbSe}_{2}$ is a more ideal platform than TI/BSCCO for the proposed novel physics if the system can be further optimized to increase the helical Cooper pairing channel by tuning the material parameters.

We discuss one of the interesting phenomena-the condensed matter supersymmetry-which can be enabled by our identification of a topological superconductor with helical-Cooper pairing ${ }^{35}$. As shown in Fig. 5c,d, magnetic doping or an external inplane magnetic field is necessary to drive the system to the critical point between the helical superconductivity and the normal Dirac gas states, with the chemical potential tuned to the Dirac point (demonstrated in Supplementary Information A.4). Under this condition, theory predicts that topological surface states (a fermionic excitation) and the fluctuations of superconducting order (a bosonic excitation) satisfy a supersymmetry relationship, and therefore, strikingly, possess the same Fermi/Dirac velocity and the same lifetime or self-energy ${ }^{4}$. We note that our present data do not provide evidence for supersymmetry, but this is an exciting future direction that we hope to access thanks to our identification of the helical-Cooper pairing and its magnetic doping response. Although the superpartners of elementary particles in high-energy physics have never been experimentally observed, the experimental methodologies, artificial sample fabrication control and experimental observations reported here pave the way for simulating and testing high-energy physics concepts in a solid-state chip $^{35}$.

\section{Methods}

Single-crystalline $2 \mathrm{H}-\mathrm{NbSe}_{2}$ samples $(\sim 3 \mathrm{~mm} \times 3 \mathrm{~mm})$ were grown using the iodine vapour transport method $^{33}$, where a high residual resistivity ratio $\left(\mathrm{RRR}=\rho_{300 \mathrm{~K}} / \rho_{0 \mathrm{~K}} \sim 100\right)$ was achieved. The $\mathrm{NbSe}_{2}$ crystals were cleaved in situ under ultrahigh vacuum, and high-quality topological insulator $\mathrm{Bi}_{2} \mathrm{Se}_{3}$ thin films were then grown on top of freshly cleaved surfaces of $\mathrm{NbSe}_{2}$ crystals using standard MBE methods (Supplementary Information B).

Ultralow temperature and ultrahigh energy resolution ARPES measurements were performed using the UE112-lowE-PGM-b+1 ${ }^{3}$ ARPES beamline in the BESSY II storage ring in Berlin, Germany, which was equipped with a VG Scienta 4000 electron analyser and ${ }^{3} \mathrm{He}$ cryo-manipulator, using an incident photon energy range from $17 \mathrm{eV}$ to $80 \mathrm{eV}$, with the lowest achievable sample temperature of about $0.9 \mathrm{~K}$ and a base pressure of $<5 \times 10^{-11}$ torr. The total energy and momentum resolution were set to $\sim 2.4 \mathrm{meV}$ and $0.01 \AA^{-1}$. Spin-resolved ARPES measurements were performed with linearly $p$-polarized light at synchrotron radiation photon energies from $50 \mathrm{eV}$ to $70 \mathrm{eV}$, where the final state effects have been demonstrated to be negligible ${ }^{34}$. Further ARPES measurements characterizing the overall electronic structure of $\mathrm{Bi}_{2} \mathrm{Se}_{3} / \mathrm{NbSe}_{2}$ (including $\mathrm{NO}_{2}$ adsorption experiments) were also performed with $29-64 \mathrm{eV}$ incident photon energies at beamlines 4.0.3, 10.0.1 and 12.0.1 at the Advanced Light Source (ALS) in the Lawrence Berkeley National Laboratory (LBNL) in Berkeley, and with a $10-30 \mathrm{eV}$ incident photon energy range at beamline 5-4 of the Stanford Synchrotron Radiation Lightsource (SSRL) in the Stanford Linear Accelerator Center (SLAC) Laboratory in Menlo Park. The base temperature and base pressure of the ARPES beamlines at the ALS and the SSRL were about $15 \mathrm{~K}$ and $<5 \times 10^{-11}$ torr, and the total energy and momentum resolution of these beamlines were about $10-15 \mathrm{meV}$ and $0.01 \AA^{-1}$, respectively.

Received 4 June 2014; accepted 22 September 2014; published online 2 November 2014

\section{References}

1. Hasan, M. Z. \& Kane, C. L. Topological insulators. Rev. Mod. Phys. 82, 3045-3067 (2010).

2. Qi, X-L. \& Zhang, S-C. Topological insulators and superconductors. Rev. Mod. Phys. 83, 1057-1110 (2011).

3. Wray, L. A. et al. Observation of topological order in a superconducting doped topological insulator. Nature Phys. 6, 855-859 (2010).

4. Grover, T., Sheng, D. N. \& Vishwanath, A. Emergent space-time supersymmetry at the boundary of a topological phase. Science 344, 280-283 (2014).

5. Nandkishore, R., Levitov, L. S. \& Chubukov, A. V. Chiral superconductivity from repulsive interactions in doped graphene. Nature Phys. 8, 158-163 (2012).

6. Fu, L. \& Kane, C. L. Superconducting proximity effect and Majorana fermions at the surface of a topological insulator. Phys. Rev. Lett. 100, 096407 (2008).

7. Qi, X-L., Hughes, T. L., Raghu, S. \& Zhang, S-C. Time-reversal-invariant topological superconductors and superfluids in two and three dimensions. Phys. Rev. Lett. 102, 187001 (2009).

8. Potter, A. C. \& Lee, P. A. Engineering a $p+i p$ superconductor: Comparison of topological insulator and Rashba spin-orbit-coupled materials. Phys. Rev. B 83, 184520 (2011).

9. Sau, J. D. et al. A generic new platform for topological quantum computation using semiconductor heterostructures. Phys. Rev. Lett. 104, 040502 (2010).

10. Hosur, P. et al. Majorana modes at the ends of superconductor vortices in doped topological insulators. Phys. Rev. Lett. 107, 097001 (2011).

11. Hor, Y. S. et al. Superconductivity in $\mathrm{Cu}_{x} \mathrm{Bi}_{2} \mathrm{Se}_{3}$ and its implications for pairing in the undoped topological insulator. Phys. Rev. Lett. 104, 057001 (2010).

12. Sasaki, S. et al. Topological superconductivity in $\mathrm{Cu}_{x} \mathrm{Bi}_{2} \mathrm{Se}_{3}$. Phys. Rev. Lett. 107, 217001 (2011).

13. Zhang, D. et al. Superconducting proximity effect and possible evidence for Pearl vortices in a candidate topological insulator. Phys. Rev. B 84, 165120 (2011)

14. Koren, G. et al. Proximity-induced superconductivity in topological $\mathrm{Bi}_{2} \mathrm{Te}_{2} \mathrm{Se}$ and $\mathrm{Bi}_{2} \mathrm{Se}_{3}$ films: Robust zero-energy bound state possibly due to Majorana fermions. Phys. Rev. B 84, 224521 (2011).

15. Sacépé, B. et al. Gate-tuned normal and superconducting transport at the surface of a topological insulator. Nature Commun. 2, 575 (2011).

16. Qu, F. et al. Strong superconducting proximity effect in $\mathrm{Pb}-\mathrm{Bi}_{2} \mathrm{Te}_{3}$ hybrid structures. Sci. Rep. 2, 339 (2012)

17. Cho, S. et al. Symmetry protected Josephson supercurrents in three-dimensional topological insulators. Nature Commun. 4, 1689 (2013).

18. Williams, J. R. et al. Unconventional Josephson effect in hybrid superconductor-topological insulator devices. Phys. Rev. Lett. 109, 056803 (2012)

19. Xu, J-P. et al. Artificial topological superconductor by the proximity effect. Phys. Rev. Lett. 112, 217001 (2014). 
20. Mourik, V. et al. Signatures of Majorana fermions in hybrid superconductor-semiconductor nanowire devices. Science 336, 1003-1007 (2012).

21. Liu, J. et al. Zero-bias peaks in the tunneling conductance of spin-orbit-coupled superconducting wires with and without Majorana end-states. Phys. Rev. Lett. 109, 267002 (2012).

22. Roy, D., Bondyopadhaya, N. \& Tewari, S. Topologically trivial zero-bias conductance peak in semiconductor Majorana wires from boundary effects. Phys. Rev. B 88, 020502(R) (2013).

23. Churchill, H. O. H. et al. Superconductor-nanowire devices from tunneling to the multichannel regime: Zero-bias oscillations and magnetoconductance crossover. Phys. Rev. B 87, 241401(R) (2013).

24. Lee, E. J. H. et al. Spin-resolved Andreev levels and parity crossings in hybrid superconductor-semiconductor nanostructures. Nature Nanotech. 9, 79-84 (2014).

25. Xu, S.-Y. et al. Hedgehog spin texture and Berry's phase tuning in a magnetic topological insulator. Nature Phys. 8, 616-622 (2012).

26. Okazaki, K. et al. Octet-line node structure of superconducting order parameter in $\mathrm{KFe}_{2} \mathrm{As}_{2}$. Science 337, 1314-1317 (2012).

27. Zhang, Y. et al. Crossover of the three-dimensional topological insulator $\mathrm{Bi}_{2} \mathrm{Se}_{3}$ to the two-dimensional limit. Nature Phys. 6, 584-588 (2010).

28. Neupane, M. et al. Observation of quantum-tunneling modulated spin texture in ultrathin topological insulator $\mathrm{Bi}_{2} \mathrm{Se}_{3}$ Films. Nature Commun. 5, 4841 (2014)

29. Blonder, G., Tinkham, M. \& Klapwijk, T. Transition from metallic to tunneling regimes in superconducting microconstrictions: Excess current, charge imbalance, and supercurrent conversion. Phys. Rev. B 25, 4515-4532 (1982).

30. Wang, E. et al. Fully gapped topological surface states in $\mathrm{Bi}_{2} \mathrm{Se}_{3}$ films induced by a $d$-wave high-temperature superconductor. Nature Phys. 9, 621-625 (2013).

31. Xu, S-Y. et al. Fermi-level electronic structure of a topological-insulator/cuprate-superconductor based heterostructure in the superconducting proximity effect regime. Phys. Rev. B 90, 085128 (2014).

32. Yilmaz, T. et al. Absence of a proximity effect in a topological insulator on a cuprate superconductor: $\mathrm{Bi}_{2} \mathrm{Se}_{3} / \mathrm{Bi}_{2} \mathrm{Sr}_{2} \mathrm{CaCu}_{2} \mathrm{O}_{8}$. Phys. Rev. Lett. 113, 067003 (2014)

33. Iwaya, K. et al. Electronic state of $\mathrm{NbSe}_{2}$ investigated by STM/STS. Physica $B$ 329-333, 1598-1599 (2003).
34. Sánchez-Barriga, J. et al. Photoemission of $\mathrm{Bi}_{2} \mathrm{Se}_{3}$ with circularly polarized light: Probe of spin polarization or means for spin manipulation? Phys. Rev. X 4, 011046 (2014).

35. Hasan, M. Z. Topological Insulators: A New State of Quantum Matter presentation at Nobel Symposium on New Forms of Matter http://www.fysik.su.se/ ardonne/nobel/ns156-program.pdf (2014).

\section{Acknowledgements}

The work at Princeton and Princeton-led synchrotron-based ARPES measurements is supported by US DOE DE-FG-02-05ER46200. MBE growth of $\mathrm{Bi}_{2} \mathrm{Se}_{3} / \mathrm{NbSe}_{2}$ samples at Penn State was supported by ARO through ARO-MURI (W911NF-12-1-0461). The point-contact measurements were supported by US DOE DE- FG02-08ER4653 (Q.L.) C.F. and M.J.G. are supported by the ONR under grant N00014-11-1-0728 and N00014-14-1-0123 and B.D. and M.J.G. are supported by the AFOSR under grant FA9550-10-1-0459. Initial materials work was supported by MRSEC Program Grant DMR-0819860. M.J.G. is supported by NSF CAREER Award ECCS 13-51871. F.C.C. acknowledges the support provided by MOST-Taiwan under project number NSC-102-2119-M-002-004. We gratefully acknowledge E. Rienks for assistance at the BESSY facility. We also thank J. Denlinger, S-k. Mo, A. Fedorov, and M. Hashimoto fo beamline support at the beamlines 4.0.3, 10.0.1, 12.0.1 at the ALS, and the beamline 5-4 at the SSRL, respectively.

\section{Author contributions}

S-Y.X., N.A., I.B., C.L., M.N., and G.B. conducted the ARPES experiments with the assistance from M.Z.H.; A.R. and N.S. performed the $\mathrm{Bi}_{2} \mathrm{Se}_{3} \mathrm{MBE}$ growth; S-H.H., R.S. and F.C. prepared the $\mathrm{NbSe}_{2}$ single crystalline samples; W.D. and Q.L. conducted the point-contact Andreev-reflection transport measurements; C.F., B.D., and M.J.G. performed theoretical calculations. M.Z.H. was responsible for the conception, overall direction, planning and integration among the research units.

\section{Additional information}

Supplementary information is available in the online version of the paper. Reprints and permissions information is available online at www.nature.com/reprints.

Correspondence and requests for materials should be addressed to M.Z.H

\section{Competing financial interests}

The authors declare no competing financial interests. 